\title{
Coffee prevents fatty liver disease induced by a high-fat diet by modulating pathways of the gut-liver axis
}

\author{
Paola Vitaglione ${ }^{1,2} *$, Giovanna Mazzone ${ }^{3}$, Vincenzo Lembo ${ }^{3}$, Giuseppe D’Argenio ${ }^{3}$, Antonella Rossi ${ }^{3}$, \\ Maria Guido $^{4}$, Marcella Savoia ${ }^{5}$, Federico Salomone ${ }^{6}$, Ilario Mennella ${ }^{1}$, Francesca De Filippis ${ }^{1,2}$, \\ Danilo Ercolini $^{1,2}$, Nicola Caporaso ${ }^{2,3}$ and Filomena Morisco ${ }^{2,3}$ \\ ${ }^{1}$ Department of Agricultural Sciences, University of Naples Federico II', Portici, Italy \\ ${ }^{2}$ Task Force on Microbiome Studies, University of Naples Federico II', Naples, Italy \\ ${ }^{3}$ Department of Clinical Medicine and Surgery, Gastroenterology Unit, University of Naples Federico II', Naples, Italy \\ ${ }^{4}$ Department of Medicine, University of Padua, Padua, Italy \\ ${ }^{5}$ Department of Biochemistry and Medical Biotechnology, University of Naples Federico II', Naples, Italy \\ ${ }^{6}$ Division of Gastroenterology, Azienda Sanitaria Provinciale di Catania, Catania, Italy
}

(Received 3 November 2018 - Final revision received 25 February 2019 - Accepted 27 February 2019)

Journal of Nutritional Science (2019), vol. 8, e15, page 1 of 11

doi:10.1017/jns.2019.10

Abstract

Coffee consumption is inversely associated with the risk of non-alcoholic fatty liver disease (NAFLD). A gap in the literature still exists concerning the intestinal mechanisms that are involved in the protective effect of coffee consumption towards NAFLD. In this study, twenty-four C57BL/6J mice were divided into three groups each receiving a standard diet, a high-fat diet (HFD) or an HFD plus decaffeinated coffee (HFD+COFFEE) for 12 weeks. Coffee supplementation reduced HFD-induced liver macrovesicular steatosis $(P<0 \cdot 01)$ and serum cholesterol $(P<0 \cdot 001)$, alanine aminotransferase and glucose $(P<0 \cdot 05)$. Accordingly, liver PPAR- $\alpha(P<0 \cdot 05)$ and acyl-CoA oxidase-1 $(P<0.05)$ as well as duodenal ATP-binding cassette (ABC) subfamily A1 $(A B C A 1)$ and subfamily G1 $(A B C G 1)(P<0 \cdot 05)$ mRNA expressions increased with coffee consumption. Compared with HFD animals, HFD + COFFEE mice had more undigested lipids in the caecal content and higher free fatty acid receptor-1 mRNA expression in the duodenum and colon. Furthermore, they showed an up-regulation of duodenal and colonic zonulin-1 $(P<0.05)$, duodenal claudin $(P<0.05)$ and duodenal peptide YY $(P<0.05)$ mRNA as well as a higher abundance of Alcaligenaceae in the faeces $(P<0.05)$. HFD+COFFEE mice had an energy intake comparable with HFD-fed mice but starting from the eighth intervention week they gained significantly less weight over time. Data altogether showed that coffee supplementation prevented HFD-induced NAFLD in mice by reducing hepatic fat deposition and metabolic derangement through modification of pathways underpinning liver fat oxidation, intestinal cholesterol efflux, energy metabolism and gut permeability. The hepatic and metabolic benefits induced by coffee were accompanied by changes in the gut microbiota.

Key words: Non-alcoholic steatohepatitis: Metabolic syndrome: Gut permeability: Gut microbiota: Polyphenols

Non-alcoholic fatty liver disease (NAFLD) is predominantly regarded as a hepatic manifestation of the metabolic syndrome. Approximately, 10-20\% of NAFLD cases develop non-alcoholic steatohepatitis $(\mathrm{NASH})^{(1)}$, and $10-15 \%$ of
NASH cases progress to cirrhosis, which dramatically increases the incidence of hepatocellular carcinoma ${ }^{(2)}$. The natural history of NAFLD associated with the high incidence of obesity and type 2 diabetes makes NAFLD the most common

Abbreviations: ABCA1, ATP-binding cassette subfamily A1; ABCG1, ATP-binding cassette subfamily G1; ACOX1, acyl-CoA oxidase 1; ALT, alanine aminotransferase; FFAR, free fatty acid receptor; HFD, high-fat diet; HFD+COFFEE, HFD plus decaffeinated coffee; LXR- $\alpha$, liver X receptor- $\alpha$; NAFLD, non-alcoholic fatty liver disease; PYY, peptide YY; SD, standard diet; ZO-1, zonulin-1.

* Corresponding author: Paola Vitaglione, fax +39081 7762580, email paola.vitaglione@unina.it 
cause of chronic liver disease in Western countries and it is predicted to become also the most frequent indication for liver transplantation by $2030^{(3)}$. In the last 10 years, the evidence that NAFLD actively contributes to aggravation of CVD, type 2 diabetes and chronic kidney disease became more and more robust. Therefore, a strong consensus was raised among medical doctors and researchers to consider NAFLD as a multisystem disease ${ }^{(3)}$. Thus, NAFLD is a worldwide health concern and medical and dietary strategies to manage and/or prevent its burden are urgent.

The aetiopathology of NAFLD involves many pathways that can be ascribed to the gut-liver axis and that regulate hepatic lipid accumulation and inflammation through the systemic metabolism, gut hormone release and the immune response. Digestion and absorption of dietary nutrients (mainly lipids and glucose), their utilisation and accumulation in the liver and in other tissues (as adipose tissue), glucose uptake and insulin sensitivity, gut microbiota composition, microbial metabolites (such as SCFA or secondary bile acids), as well as inflammatory and gut permeability status are all factors finely associated with the modulation of liver and gut health (for a detailed description of these factors, see the review by Marra \& Svegliati-Baroni $\left.{ }^{(4)}\right)$.

The discovery of risk factors and mechanisms underpinning NAFLD development and progression provides biological plausibility to the epidemiological evidence showing negative association between consumption of foods and beverages rich in bioactive compounds with antioxidant and antiinflammatory capacity (including phytochemicals and dietary fibres) and the risk of liver diseases ${ }^{(5)}$.

Coffee is one of the most commonly consumed beverages worldwide $^{(6)}$ and epidemiological evidence shows that its consumption is protective towards several diseases including liver fibrosis, cirrhosis, chronic liver disease and liver cancer ${ }^{(7)}$. Noteworthy, inverse associations between coffee consumption and markers of liver disease risk, such as serum levels of liver enzymes, were reported for both caffeinated and decaffeinated coffee by Xiao et al. ${ }^{(8)}$, while the lack of significant associations for decaffeinated coffee in the analysis of Dickson et al. ${ }^{(9)}$ could be explained by the generally modest consumption of that type of coffee and narrow range of intakes in the population. Similarly, the recent meta-analysis by Kennedy et al. ${ }^{(10)}$, including data from the few studies that specified coffee type consumed by population, showed that the increase of caffeinated and decaffeinated coffee consumption by two cups per $\mathrm{d}$ reduced the risk of hepatocellular carcinoma by 27 and $14 \%$, respectively. These observations are in line with scientific literature showing that components in coffee other than caffeine may be critical in eliciting the health benefits of the beverage (for reviews on coffee bioactive components and mechanisms underpinning benefits, see Salomone et al. ${ }^{(11)}$, Assy et al. ${ }^{(12)}$ and Alferink et al. ${ }^{(13)}$ ).

Among coffee components, particular attention has been devoted to polyphenols and melanoidins. They are considered the coffee components with the highest chances of eliciting health benefits, and also against colorectal cancer, because they can arrive, in part, at the colon, thus interacting with local microbiota ${ }^{(14)}$. Current trends in personalised nutrition point to the microbiota as one of the principal targets to modulate health ${ }^{(15)}$, but it is still underinvestigated in research focused on the health effect of coffee.

We previously investigated the individual roles of coffee polyphenols and melanoidins on antioxidant and antiinflammatory effects in the liver and blood of an animal model of NAFLD ${ }^{(16)}$. Data demonstrated that whole coffee was more effective than separated fractions to protect the liver from high-fat diet (HFD)-induced damage through a reduction in hepatic fat accumulation, systemic and liver oxidative stress and inflammation ${ }^{(16)}$.

The scientific literature indicates that gut health is implicated in the development and progression of liver diseases and very little recent evidence exists that coffee can modulate some intestinal functions ${ }^{(17)}$.

The present study aimed at clarifying the effect of coffee consumption on gut pathways implicated in NAFLD development such as intestinal and liver lipid metabolism, gut barrier functions and gut microbiota. To this purpose an animal study with mice fed an HFD and drinking water or a coffee extract as well as mice fed with a standard diet (SD) and drinking water for 12 weeks was implemented. Serum samples and liver histology were assessed in parallel with gene expression of molecular mediators of fat oxidation, cholesterol efflux, lipid digestion and energy metabolism regulation, gut permeability and composition of the gut microbiota.

\section{Materials and methods}

\section{Animals and treatments}

A sample size calculation, based on variations from a previous study ${ }^{(18)}$, showed that eight animals per treatment group would be sufficient to detect a threefold decrease in alanine aminotransferase (ALT) serum concentration with an $\alpha$ error of $0 \cdot 05$, a statistical power of $0 \cdot 8$ and two-sided testing in coffeetreated $v$. control mice.

A total of twenty-four male C57BL/6J mice, 4 weeks old, weighing $20 \pm 0.5 \mathrm{~g}$ were housed randomly in wire-bottomed cages. Animals were obtained from Harlan Italy and were maintained under controlled temperature conditions of $22 \pm 1^{\circ} \mathrm{C}$, with a $12 \mathrm{~h}$ light-dark cycle and free access to water.

Mice were divided into three groups ( $n 8$ each) and assigned to one of the following 12-week diets: (1) SD; (2) HFD; (3) HFD plus decaffeinated coffee solution (HFD+COFFEE).

The SD was a low-glycaemic control diet (TD.120455; Envigo Teklad). It contained $186 \mathrm{~g} / \mathrm{kg}$ of proteins $(19 \%$ of energy), $506 \mathrm{~g} / \mathrm{kg}$ of carbohydrates (51\% of energy), $62 \mathrm{~g} / \mathrm{kg}$ of fat ( $6 \%$ of energy), $35 \mathrm{~g} / \mathrm{kg}$ of cellulose and provided $3.3 \mathrm{kcal} / \mathrm{g}(13.8 \mathrm{~kJ} / \mathrm{g})$.

The HFD was an adjusted-energy diet (TD.06414; Envigo Teklad). It contained $235 \mathrm{~g} / \mathrm{kg}$ of protein (18\% of energy), $273 \mathrm{~g} / \mathrm{kg}$ of carbohydrates (21\% of energy), $343 \mathrm{~g} / \mathrm{kg}$ of fat (60\% of energy), $65.5 \mathrm{~g} / \mathrm{kg}$ of cellulose and provided $5 \cdot 1$ $\mathrm{kcal} / \mathrm{g}(21 \cdot 3 \mathrm{~kJ} / \mathrm{g})$.

A detailed composition of the two diets is reported in Supplementary Table S1.

Coffee-containing beverages were prepared by filtering on a filter paper (Whatman grade 113; Merck KGaA), a mix of 
boiling water and decaffeinated coffee powder (4:1, v/w) (Illy Caffè). Filtered coffee was portioned and stored at $-20^{\circ} \mathrm{C}$ until used. In a preliminary experiment, we found that the average daily consumption of solution (water or the coffee solution) was about $3.5(\mathrm{SD} 0 \cdot 3) \mathrm{ml} / \mathrm{mouse} / \mathrm{d}$. The coffeebased beverage was prepared by diluting $1.5 \mathrm{ml}$ of coffee in $100 \mathrm{ml}$ of water.

The dose of administered coffee corresponded to six cups of espresso coffee or two cups of filtered coffee for a person weighing $70 \mathrm{~kg}$. Food and energy intake as well as body weight were recorded weekly. Food intake was calculated based on the amount of food remaining from a known amount administered weekly.

At baseline, and at 2, 6 and 10 weeks of intervention, stool samples were collected, were snap-frozen in liquid $\mathrm{N}_{2}$ and stored at $-80^{\circ} \mathrm{C}$ until DNA extraction was performed.

After 12 weeks of treatment, animals fasting for $12 \mathrm{~h}$ were killed by using a lethal dose of anaesthetic and the liver, duodenum, colon and caecum with all the contents were harvested and snap-frozen until analyses. A portion of liver tissues was fixed in $4 \%$ formaldehyde and embedded in paraffin.

The experimental protocol was approved by the Ethics Committee for animal experiments at the Federico II University of Naples according to the institutional guidelines. All animals received humane care according to the criteria outlined in the National Institutes of Health Guide for the Care and Use of Laboratory Animals (National Institutes of Health Publication, eighth edition, 2011).

\section{Histology}

Sections ( $5 \mu \mathrm{m}$ thick) were obtained and stained with haematoxylin and eosin. A liver pathologist, who was blind to the intervention, performed the histological analysis, by evaluating the whole sections. Macrovesicular steatosis was assessed at low magnification $(4 \times)$ as percentage of affected hepatocyte and scored as grade 0 (between 0 and $5 \%$ ), grade 1 (between 6 and $33 \%$ ), grade 2 (between 34 and $66 \%$ ) and grade 3 $(>66 \%)$. Microvesicular steatosis was also evaluated at higher magnification $(20 \times)$ and recorded as percentage of affected cells. Necro-inflammatory lobular foci were sought and scored as present or absent.

\section{Biochemical analyses of serum samples}

Serum ALT, total cholesterol and TAG were measured using the routinely automatised assays (Reflotron Plus system from Roche Diagnostic). Liver TAG content was measured in lipid extracts from previously frozen liver tissue using a TAG quantification kit (BioVision) according to the manufacturer's protocol. Data obtained were normalised by tissue protein concentration measured by the Bradford assay (Bio-Rad).

\section{Analysis of liver and intestinal specimens}

Gene expression of PPAR- $\alpha$, acyl-CoA oxidase 1 (ACOX1), ATP-binding cassette (ABC) subfamily $\mathrm{A} 1$ (ABCA1) and liver $\mathrm{X}$ receptor- $\alpha(L X R-\alpha)$ in liver samples, of peptide YY
(PYY), free fatty acid receptor-1 (FFAR-1), FFAR-3, claudin, occludin, zonulin-1 (ZO-1), $A B C A 1$ and ABC subfamily $\mathrm{G} 1$ (ABCG1) in duodenum samples and of FFAR-1, FFAR-3 and ZO-1 in colon samples was evaluated by quantitative realtime (RT)-PCR analysis. Total RNA was extracted using an RNeasy Plus Mini Kit (Qiagen) from liver, duodenum and colon biopsies. The purity of total RNA was assessed using a NanoDrop ND-100 spectrophotometer at $260 \mathrm{~nm}$. A quantity of $2 \mu \mathrm{g}$ of total RNA was used in the first-strand cDNA synthesis by TaqMan Reverse Transcription Reagents (Applied Biosystems). The cDNA was diluted with RNase-free water for a final volume of $200 \mathrm{ml}$ and stored at $-20^{\circ} \mathrm{C}$ until used. PPAR- $\alpha, A C O X 1, P Y Y, F F A R-1$, FFAR-3, claudin, occludin, ZO-1, ABCA1, ABCG1 and $L X R-\alpha$ gene expression levels were analysed by Taq-Man Gene Expression Assays (Applied Biosystems) (Supplementary Table S2). Quantitative RT-PCR was carried out in triplicate using a pre-optimised primer/probe mixture and TaqMan Universal PCR Master Mix (Applied Biosystems) on a StepOne ${ }^{\mathrm{TM}}$ RT-PCR system in a forty-eightwell format (Applied Biosystems). The glyceraldehyde 3-phosphate dehydrogenase (GAPDH) housekeeping gene was used as an endogenous control, for normalisation of gene expression assays. The relative gene expression data were assessed by using the $\Delta \Delta^{\mathrm{Ct}}$ method. The sample values represent $\mathrm{X}$-fold differences from a control sample (given a designated value of 1) within the same experiment.

\section{Lipid composition of caecal content}

Extraction of lipids from caecal content samples was performed according to Gregory et al. ${ }^{(19)}$ with brief modifications. The extracts were analysed by liquid chomatography-high resolution MS by briefly adapting the method described by Bird $e t a l{ }^{(20)}$. Data were acquired on an Accela U-HPLC system coupled to an Exactive Orbitrap mass spectrometer (Thermo Fisher Scientific) equipped with a heated electrospray interface. A Kinetex $2 \cdot 6 \mu \mathrm{C} 18100$ A column $(100 \times 2 \cdot 1 \mathrm{~mm})$ (Phenomenex) thermostated at $45^{\circ} \mathrm{C}$ was used. The mobile phases consisted of 40:60 water-acetonitrile, $5 \mathrm{~mm}$ ammonium formate and 0.1\% formic acid (A) and 90:10 isopropanol-acetonitrile, $5 \mathrm{~mm}$-ammonium formate and $0.1 \%$ formic acid (B). A gradient elution as in Vitaglione et al. ${ }^{(14)}$ was applied. The flow rate was set at $200 \mu \mathrm{l} / \mathrm{min}$ and the injection volume was $10 \mu$ l. Acquisition was performed in both positive and negative ionisation modes, in the mass range of $m / ₹$ 120-1200. The resolving power was set to 50000 full width at half-maximum $(\mathrm{m} / z 200)$ resulting in a scan time of $1 \mathrm{~s}$. The automatic gain control was used in balanced mode $(1 \times 106$ ions); maximum injection time was $100 \mathrm{~ms}$. The interface parameters were as follows: spray voltage was at $3.5 \mathrm{kV}$ (positive mode) and $3.0 \mathrm{kV}$ (negative mode), capillary voltage $30 \mathrm{~V}$, heater temperature at $350^{\circ} \mathrm{C}$, capillary temperature at $250^{\circ} \mathrm{C}$, sheath gas at 35 arbitrary units, and auxiliary gas at 15 arbitrary units. Chromatographic data acquisition and peak integration were performed using Xcalibur software (Thermo Fisher Scientific). 
NEFA identification was obtained using ExactFinder 2.0 software (Thermo Fisher Scientific) by the application of a homemade database. Specific molecular formulas and their respective $m / z$ ratios were included in ExactFinder and the following parameters were selected: exact mass of monoisotopic ion (experimental mass tolerance accuracy less than 5 parts per million), isotopic pattern, retention time, signal:noise ratio higher than 5.

TAG were detected in positive mode as predominately $\left(\mathrm{M}+\mathrm{NH}_{4}\right)^{+}$ions and were integrated in the chromatographic region between retention times of 22-24.5 min. NEFA were detected in negative mode as $(\mathrm{M}-\mathrm{H})^{-}$ions in the chromatographic region between 3 and $7.5 \mathrm{~min}$.

\section{Gut microbiota composition}

Faecal samples (about $0.3 \mathrm{~g}$ ) were used for DNA extraction with the PowerSoil DNA Isolation kit (Mo Bio Laboratories, Inc.). The V1-V3 region of the 16S rRNA gene (about 520 bp) was amplified and PCR libraries were prepared and sequenced as previously reported ${ }^{(21)}$.

The sequences were analysed and filtered using QIIME 1.9.0 software ${ }^{(22)}$. Quality filtering, de novo operational taxonomic unit (OTU) picking and taxonomy assignment were carried out as previously described ${ }^{(23)}$. To avoid biases due to the different sequencing depths, OTU tables were rarefied to the lowest number of sequences per sample.

The 16S rRNA gene sequences produced in this study are available at the Sequence Read Archive (SRA) of the National Center for Biotechnology Information (NCBI), under accession number PRJNA434511.

\section{Statistical analysis}

All the animals (eight per group) were included in each analysis. Data are reported as means with their standard errors. Groups were compared using ANOVA followed by Tukey's multiple-comparison tests. A $P$ value of less than 0.05 was considered statistically significant.

For the faecal microbiota composition statistical analyses were carried out using $\mathrm{R}$ (https://www.r-project.org). Differential abundance of specific taxa according to diet or coffee assumption was determined by DeSeq2 ${ }^{(24)}$. Principal components analysis was carried out on the normalised abundance $\left(\log _{10}\right)$ of the microbiota at genus level (dudipca function in the $\mathrm{R}$ package made4). Permutational multivariate ANOVA (non-parametric MANOVA) based on Jaccard distance matrix was carried out by using 999 permutations to detect significant differences in the overall bacterial community composition as affected by the type of diet or coffee consumption, by using the adonis function in the vegan package.

\section{Results}

\section{Body weight and food intake}

After 12 weeks, HFD-fed mice (with or without coffee) weighed significantly more than mice fed the SD $(P<0.001$ for both groups) (Supplementary Fig. S1). Starting from week 8, HFD- and HFD+COFFEE-fed mice had different growth curves. HFD+COFFEE-fed mice gained progressively less body weight compared with HFD-fed mice $(P<0 \cdot 05)$. This difference was maintained until week 12 when HFD+COFFEE-fed mice weighed 34.8 (SE 2.5) $\mathrm{g}$ and HFD-fed mice weighed 38.4 (SE 3.0) $\mathrm{g}(P<0 \cdot 01)$. Food intake data showed that HFD- and HFD+COFFEE-fed mice had higher energy intake than SD-fed animals (386.2 (SE 7.1) and 369.2 (SE 5.8) v. 253.0 (SE 2.6) kcal (1615.9 (SE 29.7) and 1544.7 (sE 24.3) v. 1058.6 (sE 10.9) kJ); $P<0.001$ for both comparisons). Moreover, HFD+COFFEE-fed mice had higher food intake than HFD-fed mice (386.2 (SE 7.1) v. 369.2 (SE 5.8) kcal (1615.9 (SE 29.7) v. 1544.7 (SE 24.3) $\mathrm{kJ}) ; P<0 \cdot 05)$ (Supplementary Fig. S1(B)).

As concerns liver weight, data showed a reduced liver:body weight ratio in HFD+COFFEE-fed mice compared with HFD-fed mice (3.06 (SE 0.06) v. 3.47 (SE 0.15); $P<0.05$ ) and SD-fed mice (3.06 (SE 0.06) v. 3.59 (SE 0.06); $P<0.01$ ).

\section{Serum parameters and liver steatosis}

Serum total cholesterol, TAG, ALT and glycaemia are reported in Table 1. Both groups of HFD-fed mice had higher circulating concentrations of total cholesterol than SD-fed mice $(P<0.0001$ for HFD and $P=0.0097$ for HFD+COFFEE $v$. SD). However, lower concentrations were recorded in HFD+COFFEE-fed than HFD-fed mice $(P=0 \cdot 0001)$. Similarly, coffee intake reduced serum ALT in HFD-fed mice $(P=0 \cdot 0280)$. Interestingly, a significant

Table 1. Serum levels of total cholesterol, TAG, alanine aminotransferase (ALT) and glucose (Mean values with their standard errors; eight mice per group)

\begin{tabular}{|c|c|c|c|c|c|c|}
\hline & \multicolumn{2}{|c|}{ SD } & \multicolumn{2}{|c|}{ HFD } & \multicolumn{2}{|c|}{ HFD+COFFEE } \\
\hline & Mean & SEM & Mean & SEM & Mean & SEM \\
\hline Total cholesterol $(\mathrm{mg} / \mathrm{dl}) \ddagger$ & $106 \cdot 83$ & 11.32 & $238.50^{\star *}$ & $42 \cdot 30$ & $160 \cdot 63^{\star *} \dagger \dagger$ & 23.79 \\
\hline TAG (mg/dl)‡ & 103.67 & $22 \cdot 17$ & $110 \cdot 38$ & 34.72 & $110 \cdot 63$ & $20 \cdot 39$ \\
\hline $\operatorname{ALT}(\mathrm{U} / \mathrm{l})$ & 53.33 & 39.82 & $56 \cdot 88$ & 16.07 & $45.86 \dagger$ & $36 \cdot 20$ \\
\hline Glucose $(\mathrm{mg} / \mathrm{dl}) \ddagger$ & 271.9 & 4.92 & 275.5 & 6.07 & $82 \cdot 0^{* *} \dagger \dagger$ & 7.07 \\
\hline
\end{tabular}

SD, standard diet; HFD, high-fat diet; HFD+COFFEE, HFD plus decaffeinated coffee.

${ }^{* *}$ Mean value was significantly different from that for the SD-fed mice $(P<0.001$; ANOVA and Tukey's post hoc analysis).

Mean value was significantly different from that for the HFD-fed mice: $\dagger P<0.05, \dagger+P<0.001$ (ANOVA and Tukey's post hoc analysis).

$\ddagger$ To convert cholesterol in $\mathrm{mg} / \mathrm{dl}$ to $\mathrm{mmol} / \mathrm{l}$, multiply by 0.0259 . To convert TAG in $\mathrm{mg} / \mathrm{dl}$ to $\mathrm{mmol} / \mathrm{l}$, multiply by 0.0113 . To convert glucose in mg/dl to mmol/l, multiply by 0.0555 . 
reduction in blood glucose in HFD+COFFEE- $v$. SD-fed mice $(P=0.0085)$ was also observed.

Coffee consumption caused a reduction in liver steatosis induced by the HFD. Macrovesicular steatosis was scored 0 in all livers from SD- and HFD+COFFEE-fed mice, whereas in the HFD group only three cases had a score of 0 . The difference in steatosis grade between the HFD and SD and HFD+COFFEE groups was significant $(P<0 \cdot 01)$ (Supplementary Fig. S2). Microvesicular steatosis was also less severe in HFD+COFFEE- than HFD-fed mice, even if the difference between the two groups was not statistically significant. Necro-inflammatory lobular foci were seen in four of the HFD-fed mice and none of the HFD+COFFEE-fed group $(P=0 \cdot 01)$ (Fig. 1).

\section{Liver factors underpinning fat deposition}

Fig. 2 shows the gene expression of liver PPAR- $\alpha$ and $A C O X 1$ of mice in the three groups. Data showed a threefold and twofold up-regulation of PPAR- $\alpha$ and $A C O X 1 \mathrm{mRNA}$ expression, respectively, in the liver of HFD+COFFEE-fed mice compared with SD-fed animals $(P=0.0228$ and $P=0.0103)$ and with HFD-fed mice $(P=0.0160$ and $P=0.0355)$. This suggested that coffee can promote $\beta$-oxidation in the liver.

\section{Liver and intestinal factors underpinning cholesterol efflux}

Fig. 3 shows the effect of treatments on gene expression of hepatic $L X R-\alpha$ (Fig. 3(A)) and $A B C A 1$ (Fig. 3(B)) as well as duodenal $A B C A 1$ (Fig. 3(C)) and $A B C G 1$ (Fig. 3(D)). Liver LXR- $\alpha$ mRNA expression was increased by coffee consumption, while liver $A B C A 1 \mathrm{mRNA}$ expression appeared slightly increased following the HFD compared with the SD but the difference did not reach statistical significance $(P=0 \cdot 07)$. On the other hand, at intestinal level, an up-regulation of both $A B C A 1$ and $A B C G 1$ gene expression in HFD+COFFEEfed $v$. HFD-fed mice $(P=0.0164$ and $P=0.0306)$ was found; however, although the mRNA expressions were slightly increased $v$. SD-fed mice, they were not significant $(P=0.0882$ and $P=0.0895)$. These findings suggested that coffee intake can stimulate cholesterol efflux.

\section{Intestinal factors implicated in lipid sensing and energy metabolism}

Fig. 4 shows the effect of treatments on gene expression of duodenal and colonic FFAR-1 (Fig. 4(A) and (B)) and FFAR-3 (Fig. 4(C) and (D)) as well as duodenal PYY (Fig. 4 (E)). Coffee consumption up-regulated FFAR-1 in both duodenum $(P=0.021 v$. HFD and $P=0.029 v$. SD $)$ and colon tissue $(P=0.031 v$. HFD and $P=0.043 v$. SD) whereas FFAR-3 in the colon was up-regulated by the HFD independently from coffee $(P=0.005$ for HFD $v$. SD and $P=0.012$ for HFD +COFFEE $v$. SD). PYY gene expression was up-regulated in the duodenum of HFD+COFFEE- $v$. SD-fed mice $(P<$ 0.01) while no differences were recorded between HFD +COFFEE- and HFD-fed mice or HFD- and SD-fed mice.

Interestingly, the analysis of lipids in the caecum content showed that HFD+COFFEE-fed mice had 2.2 and 2.5 times higher amount of total lipids in the caecum than HFD- and SD-fed mice, respectively. Furthermore, looking at lipid composition, HFD+COFFEE-fed mice showed a NEFA:TAG ratio that was ten times lower than HFD-fed mice $(0.02 v \cdot 0.16 \%)$, thus meaning that the lipids were mainly in the form of TAG (Supplementary Fig. S3).

\section{Intestinal permeability and gut microbiota}

To establish whether coffee consumption may modulate intestinal permeability, the gene expression of the tight junctions ZO-1, occludin and claudin on intestinal specimens was assessed (Fig. 5). Significant up-regulations of duodenal and colonic ZO-1 and duodenal claudin mRNA expression with the HFD+COFFEE $v$. HFD $(P=0.035, P=0.044$ and $P=$ $0 \cdot 016$, respectively) and $v$. the $\mathrm{SD}(P=0.020)$ were found.

Conversely, occludin gene expression was down-regulated by the HFD and HFD+COFFEE $v$. the SD $(P=0.0027$ and $P=0.0006$, respectively).

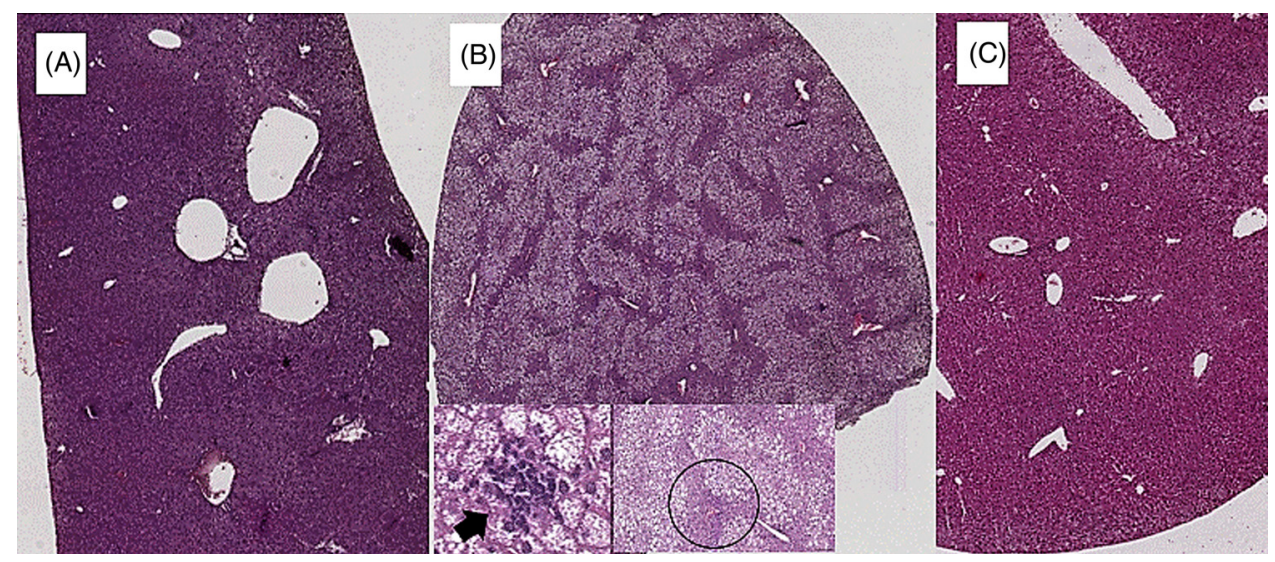

Fig. 1. Liver histology. (A) Liver from a mouse fed a standard diet showing complete absence of steatosis. (B) Severe steatosis in mouse fed a high-fat diet (HFD), only sparing a small rim of peri-portal hepatocytes (black circle); lobular necro-inflammatory foci were seen in this liver ( $\rightarrow$ ). (C) Absence of steatosis in an HFD plus decaffeinated coffee-fed mouse. 
(A)

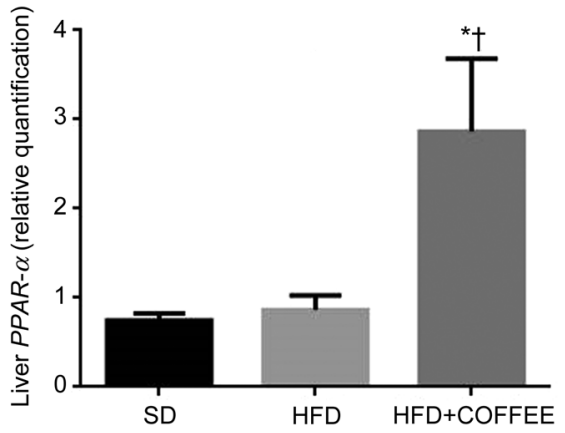

(B)

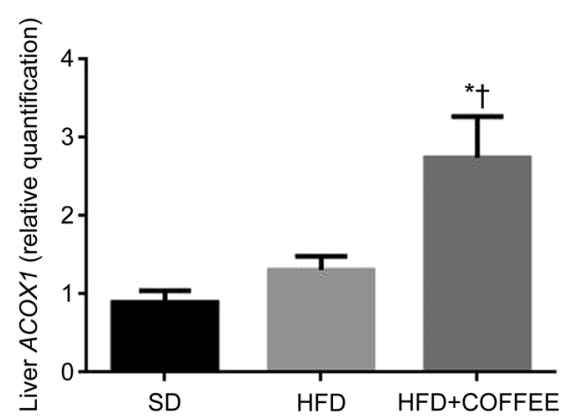

Fig. 2. Modulators of fat oxidation in the liver. Liver PPAR- $\alpha(\mathrm{A})$ and acyl-CoA oxidase 1 (ACOX1) (B) gene expression in standard diet (SD), high-fat diet (HFD) and HFD plus decaffeinated coffee (HFD+COFFEE) mice. Data are means, with standard errors represented by vertical bars. ${ }^{*}$ Mean value was significantly different from that for the SD-fed mice $(P<0.05$; ANOVA and Tukey's post hoc analysis). $†$ Mean value was significantly different from that for the HFD-fed mice $(P<0.05$; ANOVA and Tukey's post hoc analysis).

Faecal microbiota was strongly affected by the type of diet (HFD $v$. SD) $(P<0 \cdot 05)$. In particular, levels of Alistipes, Odoribacter and Allobaculum increased while Muribaculum was reduced with the HFD compared with the $\mathrm{SD}(P<0 \cdot 05)$ (Fig. 6). Interestingly, coffee intake was associated with a significant increase in Alcaligenaceae in HFD-fed mice $(0.33 \%$ in HFD+COFFEE $v .0 .03 \%$ in HFD), regardless of the length of the treatment. Principal components analysis showed a clear clustering of the samples according to diet (Supplementary Fig. S4(A)). Nevertheless, when the analysis was carried out on HFD- or SD-fed mice separately, an effect of coffee intake was observed. Samples after 6 and 10 weeks of coffee consumption (HFD+COFFEE; Supplementary Fig. S4(B)) separated from the HFD. This separation was mainly driven by the different abundance of Alistipes, Bacteroides and Odoribacter.

(A)

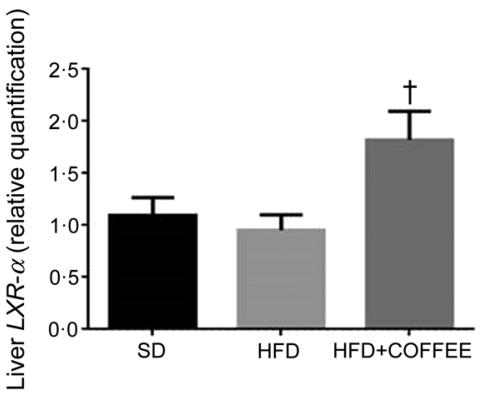

(C)

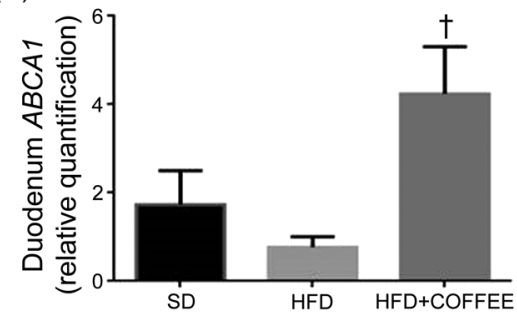

\section{Discussion}

In this study, the molecular mechanisms underpinning the effects of coffee on NAFLD induced by an HFD were elucidated by focusing on pathophysiological pathways linking the gut and liver. The main findings are summarised in Fig. 7.

Coffee consumption reduced HFD-induced liver steatosis and circulating ALT, which is consistent with our previous findings ${ }^{(16)}$. The reduced fat deposition (as demonstrated by histopathology and supported by the reduced liver:body weight ratio) and inflammation in the liver could be a consequence of an increased fat oxidation ${ }^{(25)}$, which was supported by the up-regulation of PPAR- $\alpha$. This is in agreement with other studies demonstrating the ability of polyphenols, as pure compounds or as part of foods, to induce lipid oxidation in the liver of animals fed with an $\mathrm{HFD}^{(26)}$. The hypothesis of coffee-induced fat oxidation in the liver is further supported,

(B)

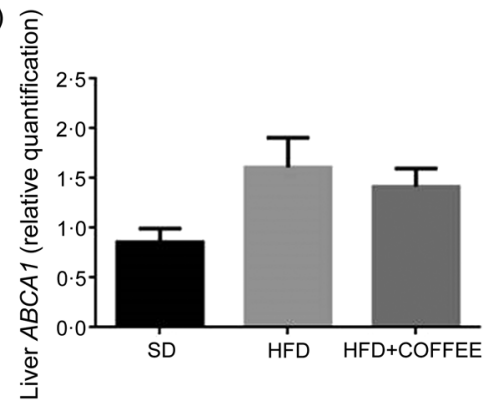

(D)

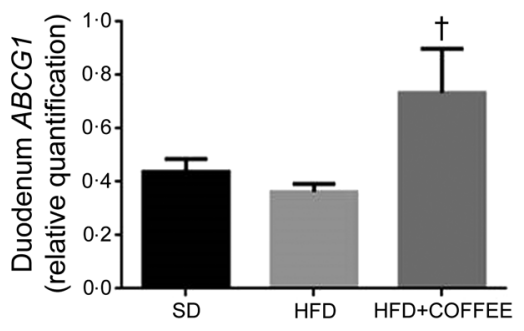

Fig. 3. Modulators of liver and intestinal cholesterol efflux. Gene expression of liver X receptor- $\alpha(L X R-\alpha)(A)$ and ATP-binding cassette subfamily $A 1$ ( $A B C A 1)$ (B) in the liver and of $A B C A 1(C)$ and ATP-binding cassette subfamily $\mathrm{G} 1$ ( $A B C G 1)(D)$ in the duodenum. Data are means, with standard errors represented by vertical bars. $\dagger$ Mean value was significantly different from that for the HFD-fed mice $(P<0.05$; ANOVA and Tukey's post hoc analysis). 
(A)

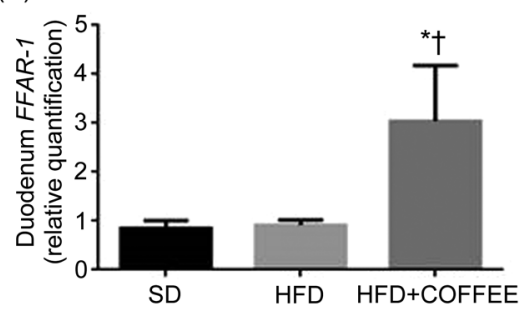

(C)

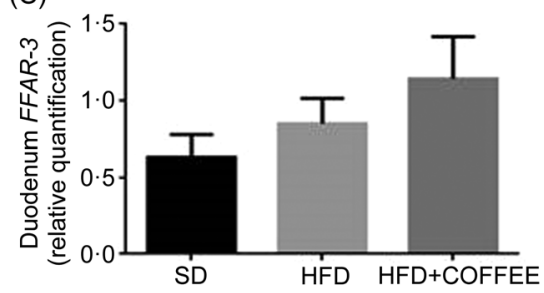

(E)

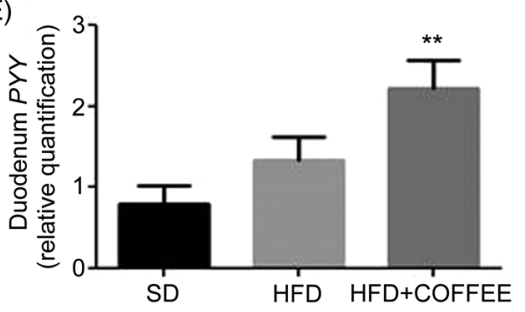

(B)

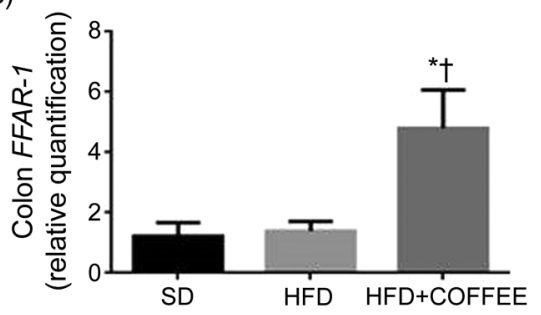

(D)

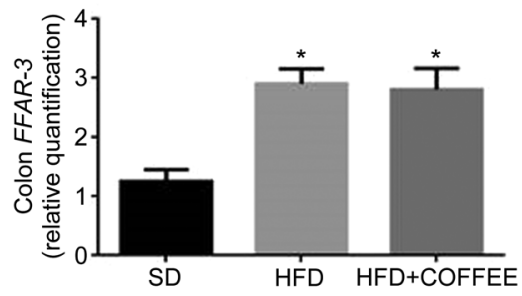

Fig. 4. Modulators of intestinal lipid sensing and energy metabolism. Gene expression of free fatty acid receptor-1 (FFAR-1) (A and B) and FFAR-3 (C and D) in the duodenum and colon, and peptide $Y Y(P Y Y)(E)$ in the duodenum of standard diet (SD), high-fat diet (HFD) and HFD plus decaffeinated coffee (HFD+COFFEE) mice. Data are means, with standard errors represented by vertical bars. Mean value was significantly different from that for the SD-fed mice: ${ }^{*} P<0.05$, ${ }^{* *} P<$ 0.01 (ANOVA and Tukey's post hoc analysis). † Mean value was significantly different from that for the HFD-fed mice $(P<0.05$; ANOVA and Tukey's post hoc analysis).

in the present study, by the up-regulation of ACOX1. Indeed, ACOX1 is responsible for the second reaction of peroxisomal $\beta$-oxidation ${ }^{(27)}$ and its up-regulation together with PPAR- $\alpha$ was a key factor in the hypolipidaemic effect of a polyphenolrich walnut extract ${ }^{(28)}$ as well as of an apple peel extract and quercetin in HFD-fed mice ${ }^{(29)}$.

(A)

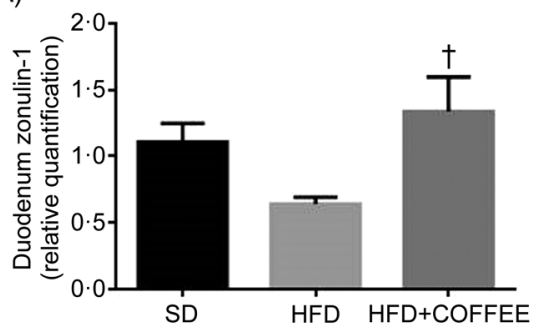

(C)

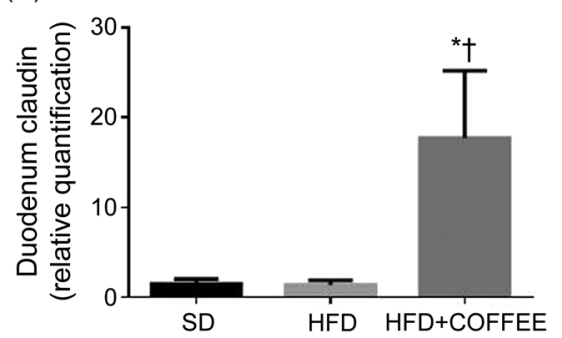

Differently from our previous study in rats ${ }^{(16)}$ coffee did not modulate serum TAG but reduced blood cholesterol and glucose in the present study. Both effects are possibly mediated by liver LXR- $\alpha$ whose activation is in line with that of PPAR $\alpha^{(30)}$. LXR- $\alpha$ could regulate systemic cholesterol homoeostasis by increasing biliary cholesterol excretion through
(B)

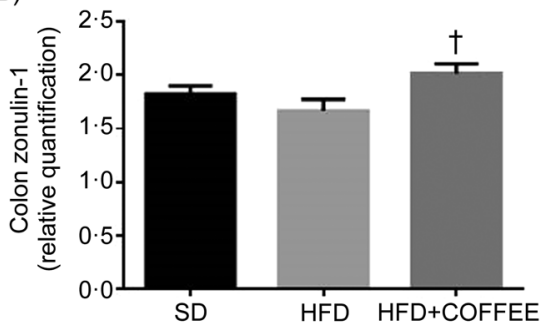

(D)

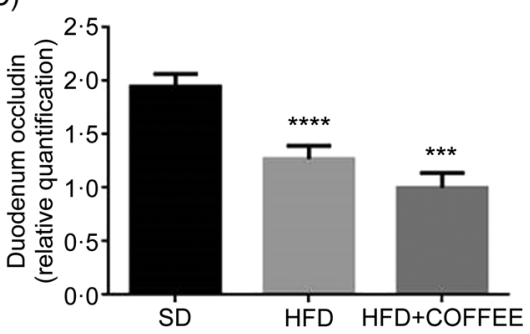

Fig. 5. Modulators of intestinal permeability. Gene expression of the tight junction proteins zonulin-1 in the duodenum (A) and colon (B), and claudin (C) and occludin (D) in the duodenum of standard diet (SD), high-fat diet (HFD) and HFD plus decaffeinated coffee (HFD+COFFEE) mice. Data are means, with standard errors represented by vertical bars. Mean value was significantly different from that for the SD-fed mice: ${ }^{\star} P<0.05,{ }^{\star \star \star} P<0.005,{ }^{\star \star \star \star} P<0.001$ (ANOVA and Tukey's post hoc analysis). † Mean value was significantly different from that for the HFD-fed mice $(P<0.05$; ANOVA and Tukey's post hoc analysis). 


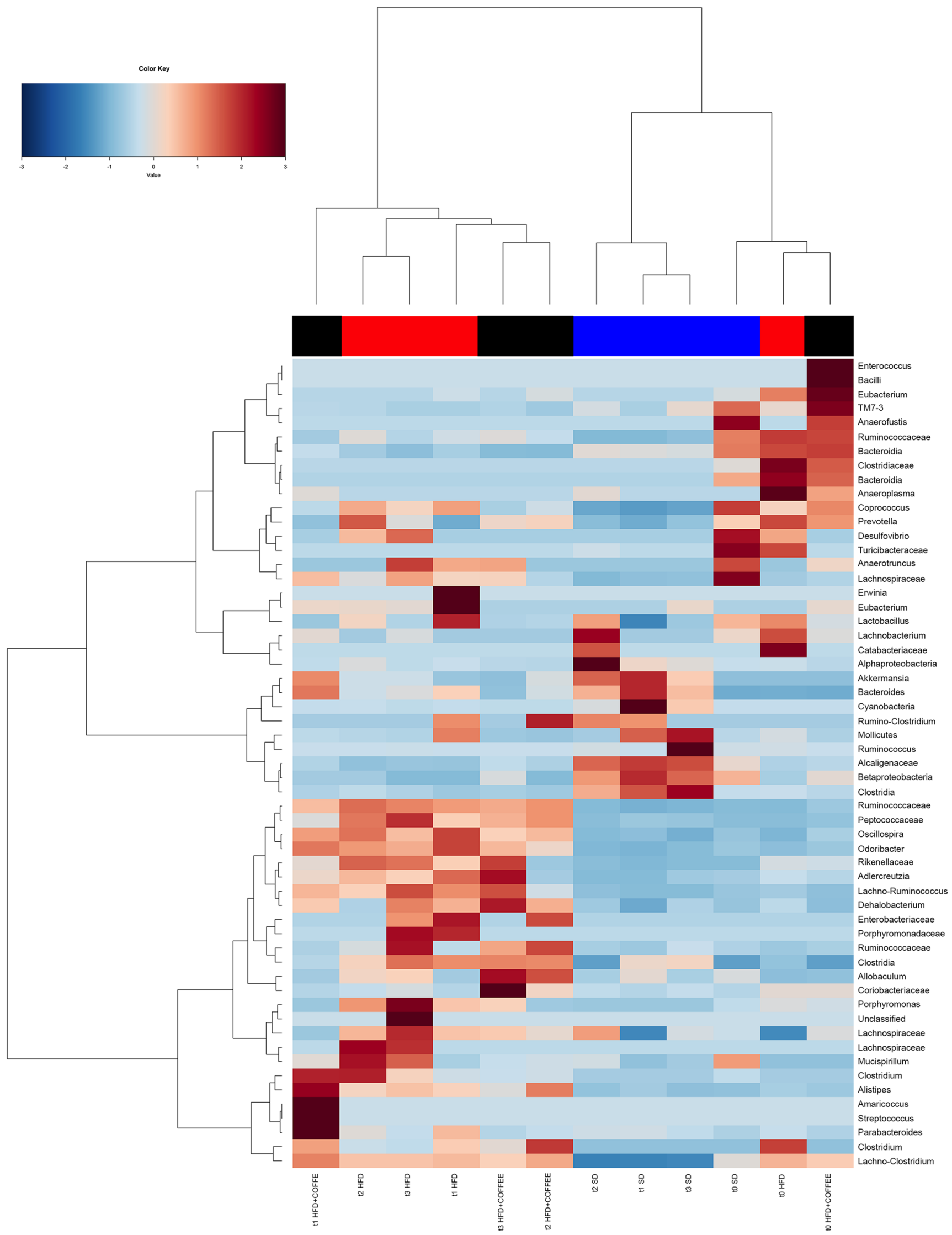

Fig. 6. Gut microbiota. Hierarchical Ward's linkage clustering of the samples based on the Pearson's correlation coefficient for the abundance of taxa present in at least $10 \%$ of the samples. The colour scale indicates the scaled abundance of each variable, denoted as the Z-score: red, high abundance; blue, low abundance. Column bars are coloured according to the dietary treatment: blue, standard diet (SD)-fed mice; red, mice fed a high-fat diet (HFD); black, mice fed an HFD and decaffeinated coffee (HFD+COFFEE). Samples are coded according to the type of diet (SD or HFD) and the length of treatment (to, baseline; t1, 2 weeks; $\mathrm{t}$, 6 weeks; $\mathrm{t} 3,10$ weeks). 


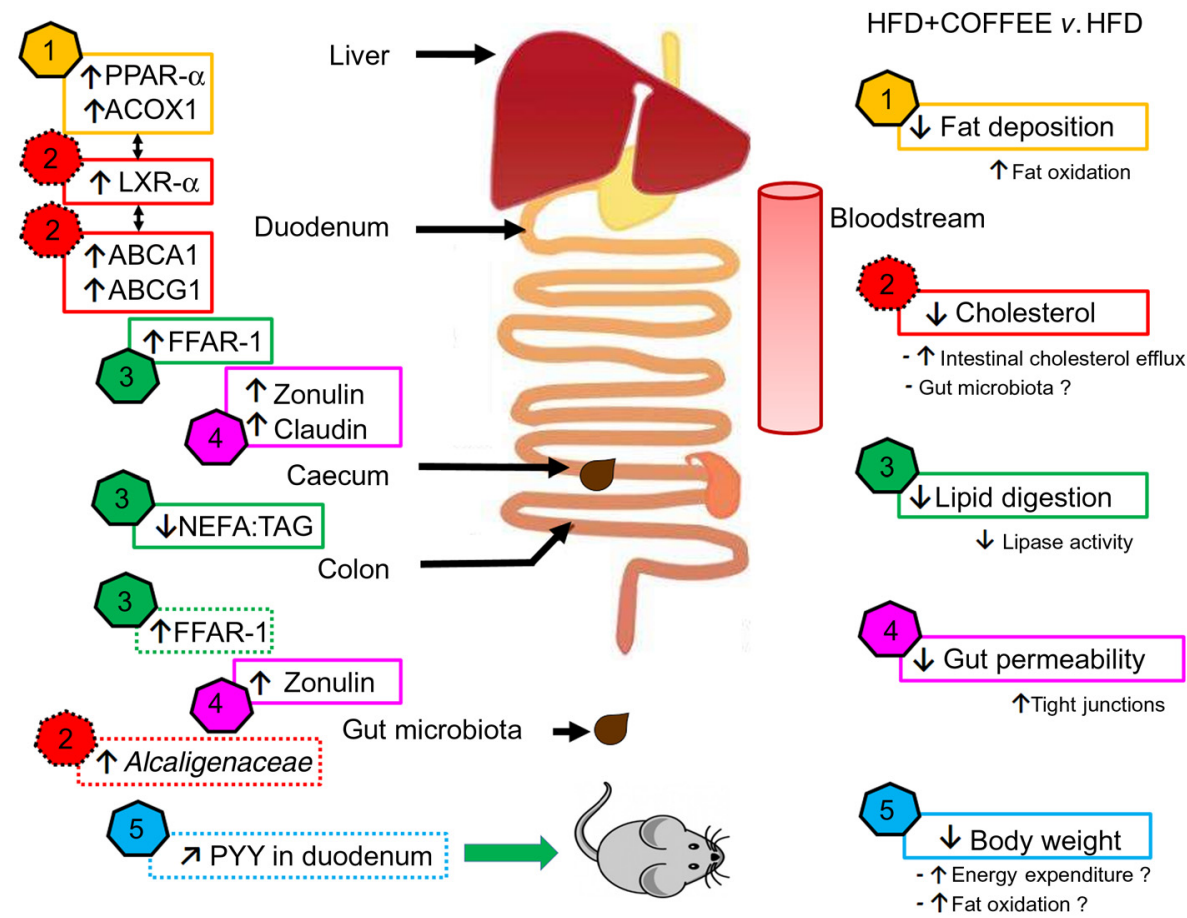

Fig. 7. Schematic representation of the main findings of the study. In the right side of the scheme, five effects of coffee consumption and the demonstrated or hypothesised (if followed by a '?') mechanisms underpinning those effects are listed. In the left side of the scheme, pathways involved at specific sites of the gastrointestinal tract are reported ( $\uparrow$, increase; $\downarrow$, reduction; $\nearrow$, slight increase but not significant). The data showed that coffee consumption determined: (1) reduction in hepatic fat deposition by increasing fat oxidation in the liver as demonstrated by up-regulation of PPAR- $\alpha$ and acyl-CoA oxidase 1 (ACOX1) gene expression; (2) reduction in circulating cholesterol by activating cholesterol intestinal efflux through up-regulation of gene expressions of liver $X$ receptor- $\alpha(L X R$ - $\alpha)$ in the liver and intestinal ATP-binding cassette subfamily A1 (ABCA1) and ATP-binding cassette subfamily G1 (ABCG1). This effect was possibly sustained by the increased abundance of Alcaligenaceae in gut microbiota; (3) reduction in lipid digestion and amelioration of the intestinal system involved in lipid sensing as demonstrated by the decreased ratio of NEFA:TAG found in the caecum content of coffee-drinking mice and by up-regulation of free fatty acid receptor-1 (FFAR-1) mRNA expression in duodenum and colon; (4) reduction in gut permeability through a restoration of tight junction proteins in the duodenum and colon as demonstrated by up-regulation of zonulin-1 and claudin gene expression; (5) control of body weight possibly through increase in energy expenditure and fat oxidation. Those conditions might be induced by an improvement in lipid sensing that possibly influenced energy metabolism regulation through amelioration of insulin sensitivity and a negligible up-regulation of intestinal peptide $Y Y(P Y Y)$ gene expression.

regulation of the intestinal membrane transporters $\mathrm{ABCA} 1$ and $A B C G 1^{(31)}$. Indeed, the two transporters act synergistically to remove cholesterol from cells: ABCA1 converts lipidpoor apoA-I to partially lipidated 'nascent' lipoproteins which are effective acceptors for cholesterol exported through the basolateral membrane by ABCG $1^{(32)}$. On the other hand, activation of LXR- $\alpha$ could also modulate liver gluconeogenesis and adipose tissue insulin-sensitive glucose transporter (GLUT4) by inducing glucose utilisation in the liver and storage in adipocytes ${ }^{(33)}$. At the intestinal level, the up-regulation of gene expression of the long-chain fatty acids receptor FFAR-1 could further support the ameliorated glucose metabolism in coffee- compared with water-drinking mice. FFAR-1 activation by NEFA in the intestinal lumen induces the secretion of glucagon-like peptide-1 and glucose-dependent insulinotropic polypeptide, which can stimulate insulin secretion glucose-dependently, thus possibly contributing to the hypoglycaemic effect elicited by coffee in HFD-fed mice ${ }^{(34)}$. Moreover, FFAR-1 activation elicits cholecystokinin response that, in turn, can stimulate secretion of pancreatic lipase and fat absorption ${ }^{(35)}$. Therefore, it was hypothesised that FFAR-1 up-regulation was an intestinal adaptive mechanism to compensate for the lacking fat digestion caused by the inhibitory activity of coffee polyphenols on pancreatic lipase ${ }^{(36-38)}$. Indeed, lipid characterisation of caecum contents showed that the NEFA:TAG ratio was lower in HFD+ COFFEE-fed mice than HFD-fed mice. An ameliorated fat absorption consequent to FFAR-1 up-regulation and possibly induced by a reinforced cholecystokinin response might provide an explanation for the similar TAG serum level in HFD- and HFD+COFFEE-fed mice despite the latter showing a reduced fat digestion.

Although statistical significance was not reached, coffee consumption slightly increased the gene expression of duodenal $P Y Y$ in HFD+COFFEE-fed mice. This observation goes in parallel with a higher food intake but lower body weight gain for HFD+COFFEE- $v$. HFD-fed mice. We hypothesised that possibly higher levels of PYY in these mice failed to increase satiety because in the presence of lipase inhibitors from coffee an attenuation of the inhibitory effect of oral fat on food intake might have occurred ${ }^{(39)}$. On the other hand, the lower body weight of coffee-drinking mice than the others lets us hypothesise that higher levels of PYY might have boosted energy expenditure and fat oxidation rate in HFD+COFFEE-fed mice ${ }^{(40)}$.

Another important finding of the present study is that coffee might have ameliorated gut permeability as suggested by the restoration of zonulin-1 gene expression in the duodenum and colon as well as claudin in the duodenum of HFD+COFFEE-fed mice $v$. HFD-fed mice. An improved 
gut barrier function induced by coffee in the present study could explain the reduced liver steatosis and the lower serum levels of cholesterol and glucose. Moreover, it is in agreement with our previous observation in rats ${ }^{(41)}$ and with the recent findings of Brandt et al. ${ }^{(17)}$ who found that consumption of decaffeinated coffee in mice fed with a highfat, -fructose and -cholesterol diet increased intestinal protein expression of zonulin-1 and occludin, reduced bacterial endotoxin concentration in portal blood and mRNA expression of lipopolysaccharide-binding protein in the liver compared with the mice fed with the same diet without coffee. It is well known that HFD is associated with dysbiosis, intestinal mucosa inflammation and a leaky gut condition leading to metabolic endotoxaemia and consequent liver fat accumulation and inflammation ${ }^{(42)}$. Prebiotics, such as inulin-type fructans, were shown to revert those conditions by modulating gut microbiota ${ }^{(4-45)}$. However, in the present study the overall structure of the gut microbiota was not significantly changed by coffee consumption, as determined by MANOVA. Nevertheless, increased abundance of Alcaligenaceae was found in mice fed the HFD and drinking coffee. Alcaligenaceae were shown to metabolise oestradiol in vitro ${ }^{(46)}$ and were negatively associated with the total cholesterol concentration in a population with atherosclerosis ${ }^{(47)}$. Therefore, it can be speculated that these bacteria might be implicated in the intestinal re-absorption of cholesterol, thus providing an upstream mechanism of cholesterol efflux to the effect of coffee on lowering serum cholesterol in the present study. A high abundance of Alcaligenaceae was also observed by Zhou et al. ${ }^{(48)}$ in mice supplemented with whole grain oat (WGO) flour and experiencing a lower blood cholesterol and improvement of insulin sensitivity in comparison with the mice supplemented with low bran oat (LBO) flour. The similar findings between the present study and the study by Zhou $e t a l .{ }^{(48)}$ could be related to the feature of WGO and coffee to be sources of antioxidant dietary fibres. Indeed, the WGO diet contained a $1.4 \%(\mathrm{w} / \mathrm{w})$ more soluble fibre (mainly $\beta$-glucans) and specific phytochemicals including phenolic acids, alkyl- and alkenylresorcinols, and avenanthramides compared with LBO. Coffee used in the present study contained melanoidins and polyphenols. Melanoidins are known to behave physiologically as dietary fibre, i.e. they can arrive undigested to the colon and can be fermented by local microbiota ${ }^{(49)}$. In addition, since melanoidins entrap polyphenols in their structure, they are chemically similar to the antioxidant cereal dietary fibres and, similarly, they behave as natural carriers of polyphenols to the colon, thus possibly beneficing colon health and nutrient metabolism through the modulation of the gut microbiota composition and functions $^{(14,50)}$.

Altogether, the mechanisms showed in our study support epidemiological data demonstrating that coffee reduces the prevalence and incidence of the metabolic syndrome ${ }^{(51)}$.

In conclusion, we provided first evidence that coffee consumption reduces HFD-induced liver damage by modulating pathways involved in the gut-liver axis.

Coffee reduced the HFD-induced liver steatosis and metabolic derangement by:
Reducing hepatic fat deposition possibly through increased fat oxidation in the liver;

Increasing cholesterol intestinal efflux;

Reducing lipid digestion and ameliorating the intestinal system involved in lipid sensing and energy metabolism regulation, and

Ameliorating gut barrier function through a restoration of tight junction proteins in the duodenum and colon.

All these mechanisms were accompanied by a negligible change in gut microbiota composition.

\section{Supplementary material}

The supplementary material for this article can be found at https://doi.org/10.1017/jns.2019.10

\section{Acknowledgements}

There are no acknowledgments.

Experiments were conducted with departmental funding. The funding source had no involvement in decisions related to the research and publication of results.

N. C., F. M. and P. V. designed the experiments, G. M., V. L., G. D. and A. R. carried out the experiments and managed animals; G. M. and M. S. performed biological analyses; M. G. and F. S. performed histology; I. M. and P. V. performed analysis of lipids; F. D. F. and D. E. performed analysis of gut microbiota; P. V., G. M. and N. C. wrote the article; P. V., N. C. and F. M. had primary responsibility for final content. All authors read and approved the final manuscript.

There were no conflicts of interest.

\section{References}

1. Hyysalo J, Mannisto VT, Zhou Y, et al. (2014) A population-based study on the prevalence of NASH using scores validated against liver histology. $J$ Hepatol 60, 839-846.

2. Ratziu V, Goodman Z \& Sanyal A (2015) Current efforts and trends in the treatment of NASH. J Hepatol 62, 1 Suppl., S65-S75.

3. Byrne CD \& Targher G (2015) NAFLD: a multisystem disease. $J$ Hepatol 62, S47-S64.

4. Marra F \& Svegliati-Baroni G (2018) Lipotoxicity and the gut-liver axis in NASH pathogenesis. J Hepatol 68, 280-295.

5. Bae M, Park YK \& Lee JY (2017) Food components with antifibrotic activity and implications in prevention of liver disease. $J$ Nutr Biochem 55, 1-11.

6. Samoggia A \& Riedel B (2018) Coffee consumption and purchasing behavior review: insights for further research. Appetite 129, 70-81.

7. Poole R, Kennedy OJ, Roderick P, et al. (2017) Coffee consumption and health: umbrella review of meta-analyses of multiple health outcomes. Br Med J 359, j5024.

8. Xiao Q, Sinha R, Graubard BI, et al. (2014) Inverse associations of total and decaffeinated coffee with liver enzyme levels in National Health and Nutrition Examination Survey 1999-2010. Hepatology 60, 2091-2098.

9. Dickson JC, Liese AD, Lorenzo C, et al. (2015) Associations of coffee consumption with markers of liver injury in the insulin resistance atherosclerosis study. BMC Gastroenterol 15, 88-97.

10. Kennedy OJ, Roderick P, Buchanan R, et al. (2017) Coffee, including caffeinated and decaffeinated coffee, and the risk of hepatocellular carcinoma: a systematic review and dose-response metaanalysis. BMJ Open 7, e013739. 
11. Salomone F, Galvano F \& Li Volti G (2017) Molecular bases underlying the hepatoprotective effects of coffee. Nutrients 9, E85.

12. Assy N, Nasser G, Kamayse I, et al. (2008) Soft drink consumption linked with fatty liver in the absence of traditional risk factors. Can J Gastroenterol 22, 811-816.

13. Alferink LJM, Kiefte-de Jong JC \& Darwish Murad S (2018) Potential mechanisms underlying the role of coffee in liver health. Semin Liver Dis 38, 193-214.

14. Vitaglione P, Fogliano V \& Pellegrini N (2012) Coffee, colon function and colorectal cancer. Food Funct 3, 916-922.

15. De Filippis F, Vitaglione P, Cuomo R et al. (2018) Dietary interventions to modulate the gut microbiome - how far away are we from precision medicine. Inflamm Bowel Dis 24, 2142-2154.

16. Vitaglione P, Morisco F, Mazzone G, et al. (2010) Coffee reduces liver damage in a rat model of steatohepatitis: the underlying mechanisms and the role of polyphenols and melanoidins. Hepatology 52, 1652-1661.

17. Brandt A, Nier A, Jin CJ, et al. (2019) Consumption of decaffeinated coffee protects against the development of early nonalcoholic steatohepatitis: role of intestinal barrier function. Redox Biol 21, 101092.

18. Matsuda Y, Kobayashi M, Yamauchi R, et al. (2011) Coffee and caffeine improve insulin sensitivity and glucose tolerance in C57BL/6J mice fed a high-fat diet. Biosci Biotechnol Biochem 75, 2309-2315.

19. Gregory KE, Bird SS, Gross VS, et al. (2013) Method development for fecal lipidomics profiling. Anal Chem 85, 1114-1123.

20. Bird SS, Marur VR, Sniatynski MJ, et al. (2011) Serum lipidomics profiling using LC-MS and high-energy collisional dissociation fragmentation: focus on triglyceride detection and characterization. Anal Chem 83, 6648-6657.

21. De Filippis F, Pellegrini N, Vannini L, et al. (2016) High-level adherence to a Mediterranean diet beneficially impacts the gut microbiota and associated metabolome. Gut 65, 1812-1821.

22. Caporaso JG, Kuczynski J, Stombaugh J, et al. (2010) QIIME allows analysis of high-throughput community sequencing data. Nat Methods 7, 335-336.

23. De Filippis F, Vannini L, La Storia A, et al. (2014) The same microbiota and a potentially discriminant metabolome in the saliva of omnivore, ovo-lacto-vegetarian and vegan individuals. PLOS ONE 9, e112373.

24. Love MI, Huber W \& Anders S (2014) Moderated estimation of fold change and dispersion for RNA-seq data with DESeq2. Genome Biol 15, 550.

25. Souza-Mello V (2015) Peroxisome proliferator-activated receptors as targets to treat non-alcoholic fatty liver disease. World J Hepatol 7, 1012-1019.

26. Salomone F, Godos J \& Zelber-Sagi S (2016) Natural antioxidants for non-alcoholic fatty liver disease: molecular targets and clinical perspectives. Liver Int 36, 5-20.

27. Reddy JK \& Hashimoto T (2001) Peroxisomal $\beta$-oxidation and peroxisome proliferator-activated receptor $\mathrm{R}$ : an adaptive metabolic system. Аnпи Rev Nutr 21, 193-230.

28. Shimoda H, Tanaka J, Kikuchi M, et al. (2009) Effect of polyphenol-rich extract from walnut on diet-induced hypertriglyceridemia in mice via enhancement of fatty acid oxidation in the liver. J Agric Food Chem 57, 1786-1792.

29. Snyder SM, Zhao B, Luo T, et al. (2016) Consumption of quercetin and quercetin-containing apple and cherry extracts affects blood glucose concentration, hepatic metabolism, and gene expression patterns in obese C57BL/6J high fat-fed mice. J Nutr 146, 1001-1007.

30. Li AC \& Glass CK (2004) PPAR- and LXR-dependent pathways controlling lipid metabolism and the development of atherosclerosis. J Lipid Res 45, 2161-2173.

31. Boullier A, Bird DA, Chang MK, et al. (2001) Scavenger receptors, oxidized LDL, and atherosclerosis. Ann N Y Acad Sci 947, 214-223.
32. Vaughan AM \& Oram JF (2006) ABCA1 and ABCG1 or ABCG4 act sequentially to remove cellular cholesterol and generate cholesterol-rich HDL. J Lipid Res 47, 2433-2443.

33. Laffitte BA, Chao LC, Li J, et al. (2003) Activation of liver X receptor improves glucose tolerance through coordinate regulation of glucose metabolism in liver and adipose tissue. Proc Natl Acad Sci U S A 100, 5419-5424.

34. Edfalk S, Steneberg P \& Edlund H (2008) Gpr40 is expressed in enteroendocrine cells and mediates free fatty acid stimulation of incretin secretion. Diabetes 57, 2280-2287.

35. Lo CM, King A, Samuelson LC, et al. (2010) Cholecystokinin knockout mice are resistant to high-fat diet-induced obesity. Gastroenterology 138, 1997-2005.

36. Cha KH, Song DG, Kim SM, et al. (2012) Inhibition of gastrointestinal lipolysis by green tea, coffee, and gomchui (Ligularia fischerr) tea polyphenols during simulated digestion. J Agric Food Chem 60, 7152 7157.

37. Murase T, Yokoi Y, Misawa K, et al. (2012) Coffee polyphenols modulate whole-body substrate oxidation and suppress postprandial hyperglycaemia, hyperinsulinaemia and hyperlipidaemia. $\mathrm{Br} J$ Nutr 107, 1757-1765.

38. Almoosawi S, McDougall GJ \& Fyfe L (2010) Investigating the inhibitory activity of green coffee and cacao bean extracts on pancreatic lipase. Nutr Bull 35, 207-212.

39. O'Donovan D, Feinle-Bisset C \& Wishart J (2003) Lipase inhibition attenuates the acute inhibitory effects of oral fat on food intake in healthy subjects. BrJ Nutr 90, 849-852.

40. Guo Y, Ma L \& Enriori PJ (2006) Physiological evidence for the involvement of peptide YY in the regulation of energy homeostasis in humans. Obesity (Silver Spring) 14, 1562-1570.

41. Mazzone G, Lembo V, D'Argenio G, et al. (2016) Decaffeinated coffee consumption induces expression of tight junction proteins in high fat diet fed rats. Funct Foods Health Dis 6, 602-611.

42. Cani PD, Bibiloni R, Knauf C, et al. (2008) Changes in gut microbiota control metabolic endotoxemia-induced inflammation in high-fat diet-induced obesity and diabetes in mice. Diabetes $\mathbf{5 7}$, 1470-1481.

43. Tilg H, Cani PD \& Mayer EA (2016) Gut microbiome and liver diseases. Gut 65, 2035-2044.

44. Cani PD, Amar J, Iglesias MA, et al. (2007) Metabolic endotoxemia initiates obesity and insulin resistance. Diabetes 56, 1761-1772.

45. Cani PD, Possemiers S, Van de Wiele T, et al. (2009) Changes in gut microbiota control inflammation in obese mice through a mechanism involving GLP-2-driven improvement of gut permeability. Gut 58, 1091-1103.

46. Payne DW \& Talalay P (1985) Isolation of novel microbial 3 $\alpha-, 3 \beta-$, and $17 \beta$-hydroxysteroid dehydrogenases. Purification, characterization, and analytical applications of a $17 \beta$-hydroxysteroid dehydrogenase from an Alcaligenes sp. J Biol Chem 260, 13648-13655.

47. Ziganshina EE, Sharifullina DM \& Lozhkin AP (2016) Bacterial communities associated with atherosclerotic plaques from Russian individuals with atherosclerosis. PLOS ONE 11, e0164836.

48. Zhou AL, Hergert N, Rompato G, et al. (2015) Whole grain oats improve insulin sensitivity and plasma cholesterol profile and modify gut microbiota composition in C57BL/6J mice. J Nutr 145, 222-230.

49. Morales FJ, Somoza V \& Fogliano V (2012) Physiological relevance of dietary melanoidins. Amino Acids 42, 1097-1109.

50. Vitaglione P, Napolitano A \& Fogliano V (2008) Cereal dietary fibre: a natural functional ingredient to deliver phenolic compounds into the gut. Trends Food Sci Technol 19, 451-463.

51. Marventano S, Salomone F, Godos J, et al. (2016) Coffee and tea consumption in relation with non-alcoholic fatty liver and metabolic syndrome: a systematic review and meta-analysis of observational studies. Clin Nutr 35, 1269-1281. 